\title{
A Molecular Dynamics Study on the Effects of Lattice Defects on the Phase Transformation from BCC to FCC Structures
}

\author{
Takuya Uehara \\ Department of Mechanical Systems Engineering, Yamagata University, Yonezawa, Japan \\ Email: uehara@yz.yamagata-u.ac.jp
}

How to cite this paper: Uehara, T. (2019) A Molecular Dynamics Study on the Effects of Lattice Defects on the Phase Transformation from BCC to FCC Structures. Materials Sciences and Applications, 10, 543557.

https://doi.org/10.4236/msa.2019.108039

Received: July 12, 2019

Accepted: August 13, 2019

Published: August 16, 2019

Copyright () 2019 by author(s) and Scientific Research Publishing Inc. This work is licensed under the Creative Commons Attribution International License (CC BY 4.0).

http://creativecommons.org/licenses/by/4.0/

\begin{abstract}
Molecular dynamics simulations of the phase transformation from bodycentered-cubic $(\mathrm{bcc})$ to face-centered-cubic $(\mathrm{fcc})$ structures were performed. A Morse-type function was applied, and the parameters were determined so that both fcc and bcc structures were stable for the perfectcrystal model. When the fcc structure was superior to the bcc structure, the bcc model transformed to fcc. Two mechanisms, based on the Bain and NishiyamaWasserman (NW) relationships, were considered. Then, point or linear lattice defects, i.e., randomly scattered or regularly aligned vacancies, were introduced. Consequently, bcc models tended to transform to an fcc structure, whereas fcc models remained stable. The transformation process was also investigated in detail. BCC-to-FCC transformation is often considered as a homogeneous process based on changes in the axis lengths, and such a process was observed for the perfectcrystal model. Conversely, for the defect models, local heterogeneous deformation patterns, including cylindrical domain and planar interface formation, were observed. These behaviors are considered to be related to plastic deformation during phase transformation, and the validity of the presented model for further investigation was confirmed.
\end{abstract}

\section{Keywords}

Molecular Dynamics, Phase Transformation, Lattice Defect, Plastic

Deformation, Transformation Plasticity

\section{Introduction}

The microstructure of materials strongly affects the macroscopic mechanical properties, and controlling the microstructure is important for achieving re- 
quired properties. With steel, heat treatment processes such as quenching, tempering, and annealing are the most effective routes that are indispensable for producing useful materials [1]. This process involves intrinsic phase transformations between austenite, ferrite, and martensite; austenite phase is stable at high temperatures, with a face-centered-cubic (fcc) structure, while ferrite and martensite phases are stable or quasi-stable at room temperature with body-centered-cubic (bcc) and body-centered-tetragonal (bct) structures, respectively. Heat treatment processes are classified into many detailed subdivisions, and the obtained phases are named as pearlite, bainite, troostite, and so on, while the fundamental crystal structures are considered to be fcc and bcc/bct. The development of heat treatment processes has a long history, but advancement has been achieved through trial and error based on enormous experiments. Additionally, the precise control of not only the microstructure but also the deformation and stress distribution has been required. In addition to distortion due to phase transformation, plastic deformation plays a substantial role in deformation and is termed transformation plasticity. However, even the fundamental mechanism remains unclear, and intensive investigations involving modeling the phenomena and clarifying the mechanism have been conducted [2]-[7].

Alternatively, computer simulation is an indispensable tool in engineering processes. In heat treatment processes of steel, it has become possible to simulate macroscopic deformation and stress distribution, where the phase transformation is usually represented by an empirical kinetic law such as the JohnsonMehl-Avrami-Kolmogorov equation, and the state of a phase is normally represented using the volume fraction of eachphase [8] [9]. From a smaller-scale viewpoint, it has become possible to simulate the microstructure using a phase field model [10] [11], in which two phases are represented by a single variable with a value of 0 or 1 , and phase transformation is expressed by the domain boundary motion. However, these computational simulations are phenomenological, and the essential phenomena, i.e., change in the crystal structure or atomic configuration, are not directly represented. In order to achieve an intrinsic understanding and application through more detailed and precise modeling and simulation, atomistic investigations are necessary.

Experimental technology on a nanometer scale has achieved rapid progress using electron and atomic-force microscopy [12] [13], however, direct observation of dynamic behavior is not yet possible. Molecular dynamics (MD) simulation is useful for exploring the atomic behavior, and various researches including on the structural phase transformation have been carried out [14]-[19]. However, few studies on the mechanism of the transformation plasticity are reported. In this study, we modeled the phase transformation on an atomic scale to clarify the deformation mechanism during phase transformation, including induced plastic behavior. In MD modeling, a choice of potential function is crucial, and this should be carefully demonstrated when a specific material is studied. In this study, we concentrated on capturing a general aspect of the phase 
transformation between bcc and fcc structures, and a Morse-type potential, for which parameters for both fcc and bcc materials are listed [20], was applied. The parameter was determined initially so that both fcc and bcc structures are stable, and then the effects of lattice defects on the transformation process were investigated. Three types of defects, i.e., point, partial line, and full-line defects, were considered, though these are all based on vacancies, and their influence on the stable phase was investigated. Generation of plastic behavior during phase transformation was also investigated by observing the dynamic process.

\section{Crystallographic Relationship between Bcc and Fcc}

The crystallographic relationship between the fcc and bcc structures is usually explained as the Bain relationship. Figure 1(a) shows a bcc-based schematic; four bcc unit cells are illustrated by green lines, and the corner and body-centered atoms are depicted in orange/yellow and blue/light blue, respectively. In the bcc lattice, another unit cell is drawn using black lines, in which atoms are positioned at the eight corners and the center of each face of the unit cell. The top and bottom faces are square with a length of $\sqrt{2} L$, where $L$ is the edge length of the original bcc unit cell, whereas the side faces are rectangles with the lengths of $L$ and $\sqrt{2} L$ in the vertical and horizontal directions, respectively. This structure corresponds to a face-centered-tetragonal (fct). If the length of the vertical axis ( $z$ in Figure 1$)$ is increased by $\sqrt{2}$, or the two perpendicular axes are shortened by $1 / \sqrt{2}$, the cell becomes cubic. Accordingly, the phase transformation from bcc to fcc can occur only by changing the edge length without changing the atomic arrangement.

Another relationship between fcc and bcc structures is Nishiyama-Wasserman (NW) relationship, as shown in Figure 1(b). This is usually used to explain martensitic (bct) phase transformation, which is interpreted as a bcc-fcc relationship when combined with a change in the edge length [21]. The crystal plane and orientation are related through fcc $\{111\} / / \mathrm{bcc}\{011\}$ and fcc $<112>/ / \mathrm{bcc}$ $<011>$. In Figure 1(b)(i), two bcc unit cells are drawn using green lines, two parallel (011) planes are drawn using black and red lines, and the $[01 \overline{1}]$ direction is indicated by an arrow in magenta. Figure 1(b)(ii) shows the atomic arrangement from the direction perpendicular to the (011) plane. Here the black and red rectangles in (i) are seen with the half-cell offset. In this plane, a hexagon is drawn in blue. From this arrangement, if the atoms in the red rectangular plane move in the $[01 \overline{1}]$ direction, and if the edge length in the corresponding direction is slightly elongated so that the hexagon becomes regular, then this plane becomes the (111) plane with appropriate stacking, and the bcc-to-fcc transformation is complete. This mechanism requires both the change in edge length and relative motion of the atomic layer, but the required edge-length ratio is much smaller than that for the Bain relationship. Figure 1(c) illustrates, for 


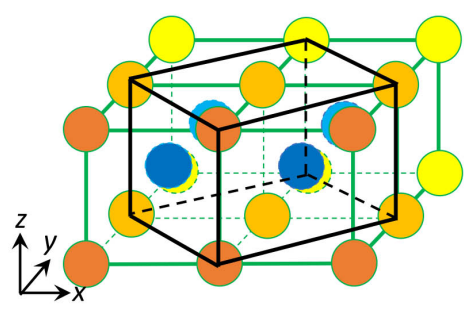

(a) Bain relationship

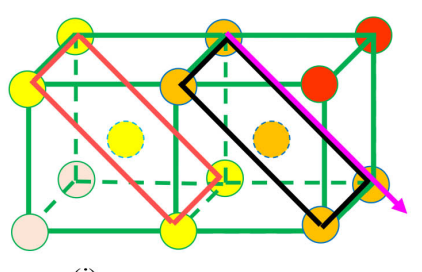

(i)

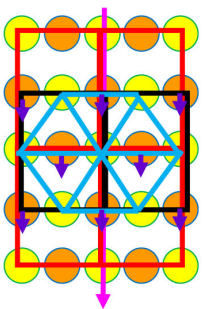

(ii)

(b) NW-based relationship
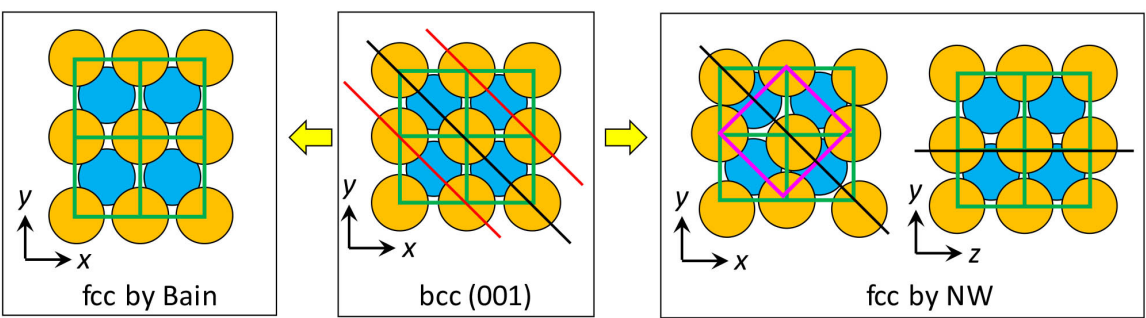

(c) Atomic arrangement on the (001) plane before and after bcc-to-fcc transformation

Figure 1. Crystallographic relationship between bcc and fcc structures.

visualization of the expected results, the atomic configuration projected on the $x-y$ and $z-y$ planes when the bcc-to-fcc transformation occurs. The original (001) plane becomes the (011) plane of the fcc structure, following the Bain relationship, and a rectangular cell is prominent. However, relative motion of alternate layers, represented by black lines, occurs in the NW relationship, giving a somewhat disordered impression. The regular unit can be found by selecting a $45^{\circ}$ rotated square, depicted in magenta, in which the center atom is shifted to one side. In the perpendicular $(z-y)$ plane, the long and short edged rectangular cells are alternatively accumulated along the $z$ direction.

\section{Molecular Dynamics Method}

A classical MD method with two-body interatomic potential function was used in this study, and the following Newton equation of motion was then solved numerically.

$$
\frac{\mathrm{d}^{2} r_{i}}{\mathrm{~d} t^{2}}=\frac{1}{m_{i}} \boldsymbol{F}_{i}=\frac{1}{m_{i}} \sum_{j} \boldsymbol{f}_{i j}, \quad f_{i j}=-\frac{\mathrm{d} \phi}{\mathrm{d} r} \frac{r_{i j}}{\left|r_{i j}\right|}
$$

Here, $r_{i}$ and $m_{i}$ are the position vector and mass of the $i$-th atom, $F_{i}$ is the force acting on the $i$-th atom, $f_{i j}$ is the interatomic force between the $i$-th and $j$-th atoms, and $\phi$ is the interatomic potential energy function. In this study, the following Morse-type function is applied.

$$
\phi=D[\exp \{-2 \alpha(r-R)\}-2 \exp \{-\alpha(r-R)\}]
$$

Here, $D, R$, and $\alpha$ are the parameters, and the values for most major metallic elements are listed in the literature [20]. Equation (2) is standardized by dividing $\phi$ by $D$ and $r$ by $R$ as 


$$
\phi^{*}=\exp \left\{-2 \alpha^{*}\left(r^{*}-1\right)\right\}-2 \exp \left\{-\alpha^{*}\left(r^{*}-1\right)\right\} .
$$

In this paper, all variables are expressed as the standardized values, except for temperature, and the superscript ${ }^{*}$ is omitted. Numerical integration was performed using the conventional velocity Verlet algorithm.

\section{Preliminary Simulation Representing Stable Structures}

\subsection{Simulation Model and Conditions}

As a preliminary study, the potential parameter $\alpha$ was varied, and the stable structures were explored using the models illustrated in Figure 2. The atoms are arranged on lattice points of fcc or bcc structures, and periodic boundary conditions are imposed in all the directions. No defects are introduced in this section. The models shown in Figure 2(a) and Figure 2(b) have fcc and bcc structures, respectively, in both of which the (001) plane and [001] direction are set on the $x-y$ plane and along the $z$-axis. Additionally, another orientation model is prepared so that the (011) plane and [011] direction become the $x-y$ plane and $z$-axis, respectively, as shown in Figure 2(c). This crystal cell corresponds to the rectangular parallelepiped illustrated in Figure 1(a). Models (b) and (c) are essentially the same but are applied to simulations since the result can be affected by restriction of the periodicity of the calculation cell. Also, the visibility of the transformation mechanism is increased by directly observing the $\{011\}$ planes of the original bcc structure. The model size is 16-16-16 unit cells in the $x-y-z$ directions for the bcc-(001) model, and 12-12-12 for bcc-(011) model and fcc models. The difference in the size is based on the number of atoms in a unit cell to construct each model, and these sizes were determined for the total number of atoms to be close to each other (8192 and 6192, respectively).

Using these models, MD simulations were demonstrated at a constant temperature under a constant pressure, $p=0$. The velocity scaling method was applied for temperature control with $T=10,400$, and $800 \mathrm{~K}$. Total time step was set as 5000 steps, which may not be long enough to distinguish an actual stable structure, but this does not matter in this study because the transformation is the major objective.

\subsection{Results}

Figure 3 and Figure 4 represent typical results. Figure 3(a) and Figure 3(b) show snapshots of the atomic configurations at the initial and final time steps for $\alpha=1.50$ and $T=10 \mathrm{~K}$, and $\alpha=1.6$ and $T=10 \mathrm{~K}$, respectively. For the fcc model, change in the atomic configuration was not observed, including with all other parameters. For the bcc models, no change occurred for $\alpha=1.50$, whereas drastic changes occurred for $\alpha=1.60$. Figure 3(b)(i) shows the atomic configuration projected on the $x-y$ and $z-y$ planes at the 5000th time step, and Figure 4(a) shows the variation in the edge lengths and potential energy, calculated for the 


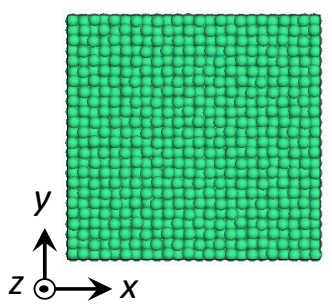

(a) fcc (001)

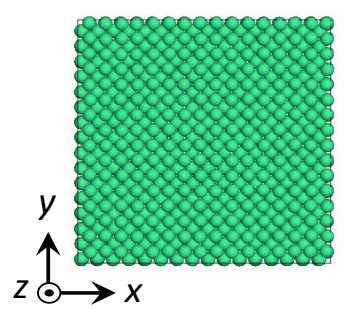

(b) bcc (001)

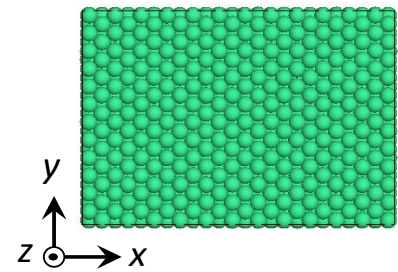

(c) bcc (011)

Figure 2. Illustration of the simulation models.

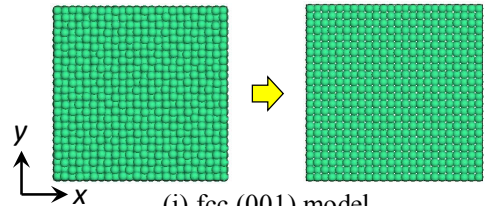

(i) fcc (001) model

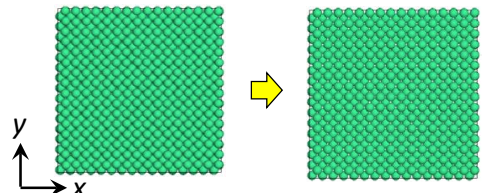

(ii) bcc (001) model

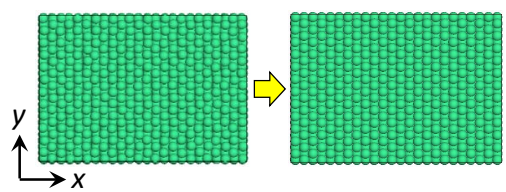

(iii) bcc (011) model

(a) $\alpha=1.50, T=10 \mathrm{~K}$

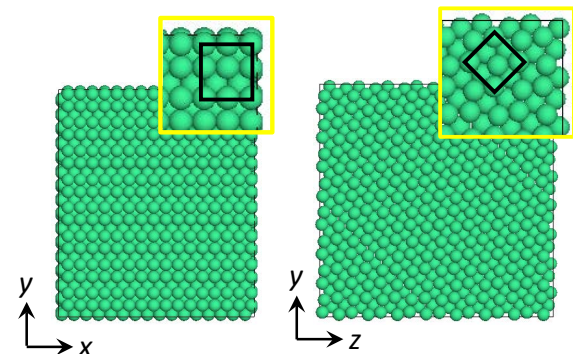

(i) bcc (001) model
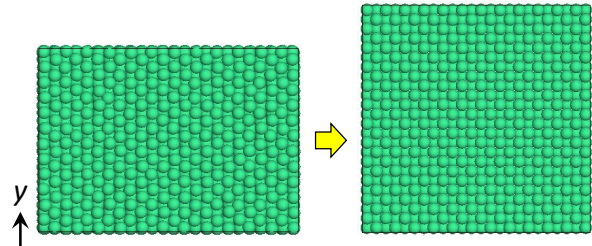

(ii) bcc (011) model

(b) $\alpha=1.60, T=10 \mathrm{~K}$

Figure 3. Stable structures obtained with several parameters and temperatures.

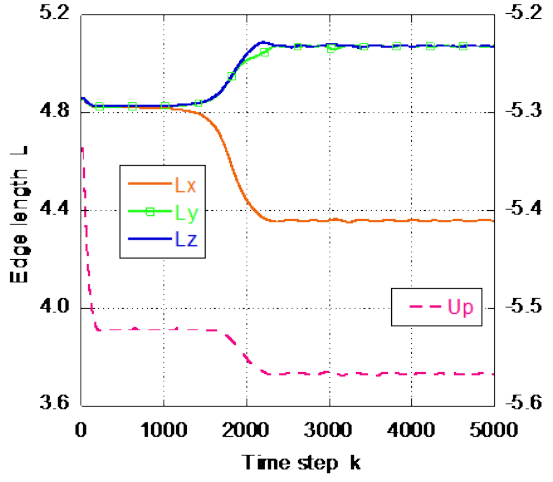

(a) $\alpha=1.50, T=10 \mathrm{~K}$

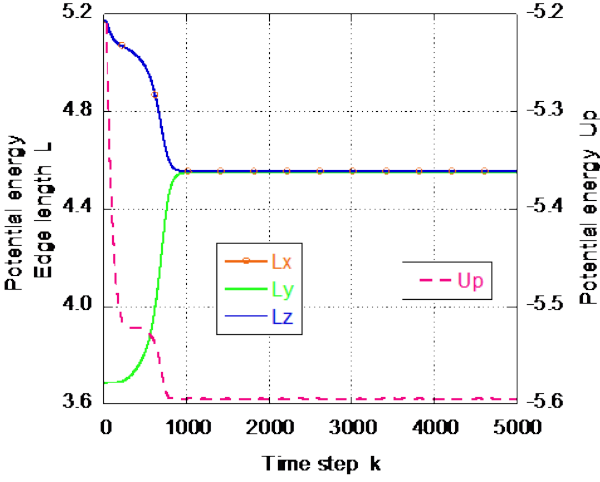

(b) $\alpha=1.60, T=10 \mathrm{~K}$

Figure 4. Variation in the edge lengths and potential energy.

bcc-(001) model. The model is initially cubic, and three edges have the same lengths. However, the $x$-edge is shortened, and the $y$ and $z$ edges are elongated between the 1000th and 2000th time steps. Consequently, the calculation cell becomes rectangular. The atomic configurations, shown in the magnified windows, correspond to those illustrated in Figure 1(c), and the edge-length ratio 
indicated in Figure 4(a) also approximately coincides with the ideal value. Therefore, it is confirmed that phase transformation from bcc to fcc has occurred according to the NW relationship.

Similarly, an fcc transformation occurred for the bcc-(011) model. The initially rectangular calculation cell became cubic by stretching the shorter edge ( $y$ in this case), as shown in Figure 3(b)(ii) and Figure 4(b). The relative configuration of atoms did not change; therefore, it is concluded that an fcc transformation occurred in accordance with the Bain relationship. In both cases, the potential energy was decreased; hence, the fcc structure is more stable for $\alpha=1.60$. Note that the difference in the potential energy between Figure 4(a) and Figure 4 (b) is due to the use of a different value in $\alpha$.

The simulated results are summarized in a phase diagram shown in Figure 5 (a), in which, for example, the notation " $\mathrm{fcc}->\mathrm{fcc}$ " indicates that the fcc model maintained its structure, and "bcc- $>\mathrm{fcc}$ " indicates that phase transformation from bcc to fcc occurred. The fcc structure was stable for the whole parameter range shown in the diagram, and no plots for "fcc->bcc" exist. It is concluded that both the fcc and bcc structures are stable or quasi-stable in the range of $\alpha \leq$ 1.55, while the bcc structures transform to fcc for $\alpha \geq 1.60$. Figure 5(b) represents the potential energy obtained for fcc and bcc structures at $T=10 \mathrm{~K}$. Note that the potential energies of bcc for $\alpha \geq 1.60$ are measured before the transformation to fcc occurred. The potential energy for fcc phase is slightly lower than that for bcc; consequently, the fcc structure is superior to bcc, and the bcc is quasi-stable for small values in $\alpha$. The difference in the potential energy becomes larger as the value in $\alpha$ becomes larger. Then the bcc phase is unable to retain the structure, and transformation occurs for $\alpha \geq 1.60$.

\section{Effects of Defects on Phase Transformation}

\subsection{Simulation Models and Conditions}

Based on the results for perfect crystal models, the models with lattice defects were applied to the MD simulations. Models based on a bcc-(001) model are shown in Figure 6. The color indicates the potential energy of each atom, and the stable atoms in a bulk crystal are depicted in blue, while those around the defects are depicted in other colors. Three types of defects, i.e., point, partial line, and full-line defects, are introduced. It should be noted that all defects here consist of vacancies; in a point model, vacancies are randomly scattered, and the proportion of vacancies is set as $5 \%$ or $10 \%$ of the lattice points. In a line-defect model, the vacancies are aligned on a straight line, and the length is set as $2 L_{a}$ or $3 L_{a}$, where $L_{a}$ is the lattice parameter. In a full-line model, the vacancy line traverses the model, resulting in an infinite line through the periodic boundary. The defect line is set in the center of the $x-y$ plane, as shown in Figure 6(b) and Figure 6(c). In this paper, these models are referred to as Point-5, Point-10, Line-2, Line-3, and Line-full models. 


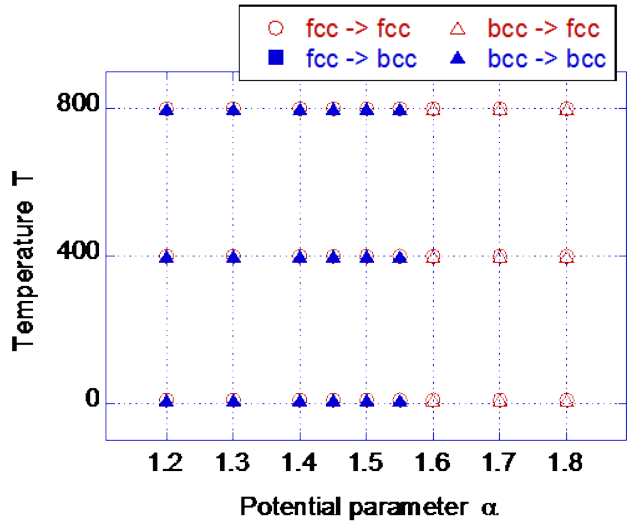

(a) Phase diagram

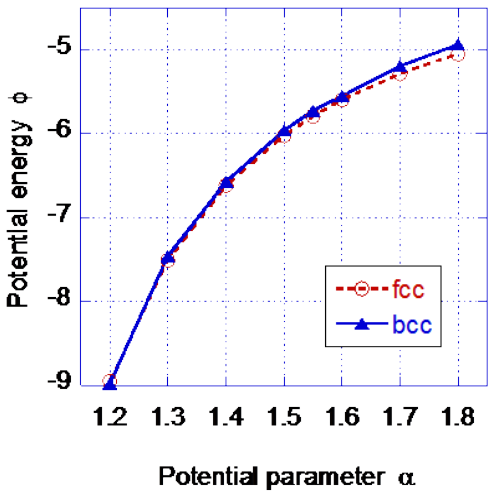

(b) Potential energy

Figure 5. Phase diagram obtained using perfect crystal model and potential energy for bcc and fcc structures at $T=10 \mathrm{~K}$.

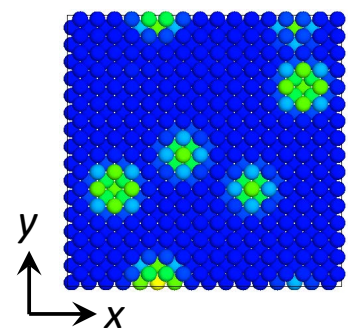

(a) Point

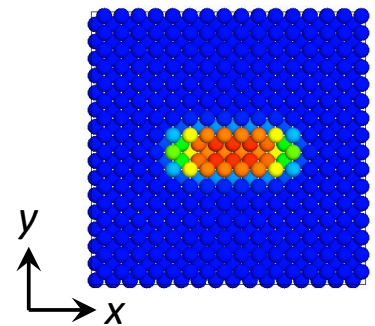

(b) Partial line

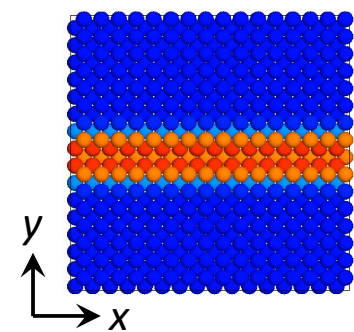

(c) Full line

Figure 6. Illustration of defect models.

According to the phase diagram in Figure 5(a), both fcc and bcc structures are stable for $\alpha \leq 1.55$. In this section, similar simulations are performed using the defect models, and the effects on the stable structure are examined. The simulation conditions are the same as in the previous section; $\alpha$ is set between 1.20 and 1.80, $T=10,400$, and $800 \mathrm{~K}$, and the initial configuration is based on fcc, bcc-(001), and bcc-(011) models. Three trials are demonstrated for each parameter set by varying the random-number series, which is used for defining the initial velocity of every atom and is considered to influence the results to a certain degree.

\subsection{Stable Structures for Defect Model}

Figure 7 represents the change in the configuration of atoms in the initial and final time steps for typical cases. For the bcc-(001) model, Point-10 and Line-3 models are shown in Figure 7(a)(i) and Figure 7(ii), respectively. No transformation was observed for $\alpha=1.50$ in the perfect crystal model, but fcc transformation was observed here. Both structures show the characteristic arrangement of the NW relationship as represented in Figure 1(c). Similarly, the bcc-(011) models also show an fcc transformation for $\alpha \leq 1.55$ with the defect models, and the transformation path was the same as for the Bain relationship with the 


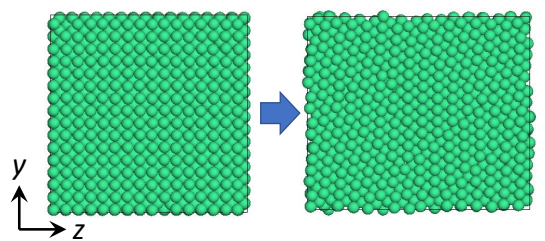

(i) $\alpha=1.50$, Point 10

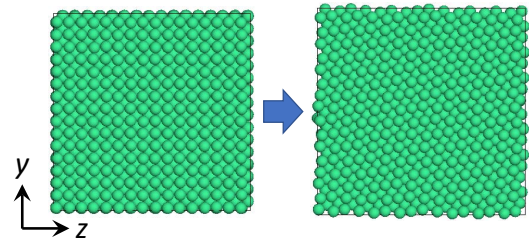

(ii) $\alpha=1.50$, Line 3

(a) bcc (001) model, $T=10 \mathrm{~K}$

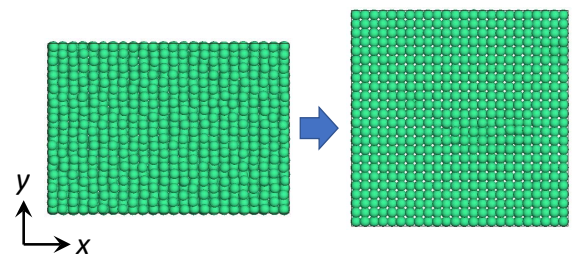

(i) $\alpha=1.55$, Point 10

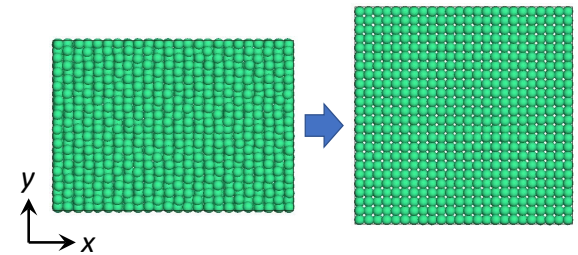

(ii) $\alpha=1.50$, Line 3

(b) bcc (011) model, $T=10 \mathrm{~K}$

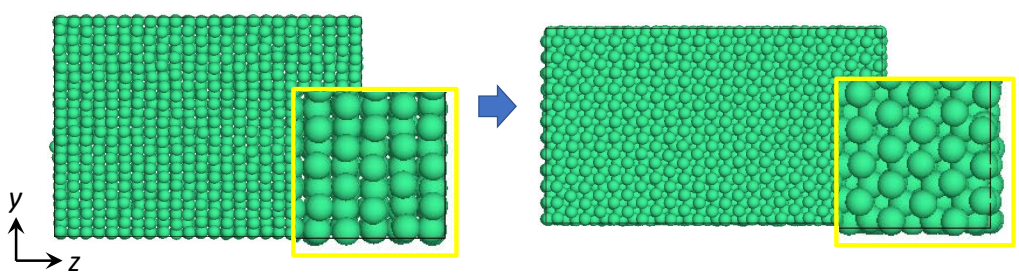

(c) bcc (011) model, $T=800 \mathrm{~K}\left(\alpha=1.55\right.$, Line $\left.2 L_{a}\right)$

Figure 7. Stable structures obtained for defect models.

perfect crystal models. Typical results for $\alpha=1.55$ with Point-10 and $\alpha=1.50$ with Line-3 models are shown in Figure 7(b)(i) and Figure 7(b)(ii), respectively.

Figure 7(c) represents a special case for a bcc-(011) model with $\alpha=1.55$, Line-2 defect, and $T=800 \mathrm{~K}$. In this case, the relative motion of the alternate layer occurred in the manner illustrated in Figure 1(c) following the NW relationship. Consequently, a complete (111) plane is observed, as clearly shown in the magnified window in Figure 7(c).

These results are summarized in a phase diagram in Figure 8(a), in which the small marks represent the results for a perfect crystal. The results for the defect models were only plotted when a bcc-to-fcc transformation occurred. Three trials were executed for each case, and the case was plotted if transformation occurred one or more times. The results for Point- 5 and Point- 10 models, and Line- 2 and Line- 3 models showed similar trend, and their results are plotted together. This figure clearly shows that the domain where the fcc structure is stable is extended, and the threshold value of $\alpha$ is decreased from 1.60 to 1.45. That is, the defects accelerate phase transformation from bcc to fcc structures. Figure 8(b) represents the potential energy in the defect models for $\alpha=1.40$ and $T=10$ $\mathrm{K}$, in which the value is the average of all atoms in the calculation cell. Blue and red lines represent the value for the perfect bcc and fcc crystal models, respectively. The potential energy for defect models is increased, but the increment is small compared to the difference between fcc and bcc. Point-defect models show 


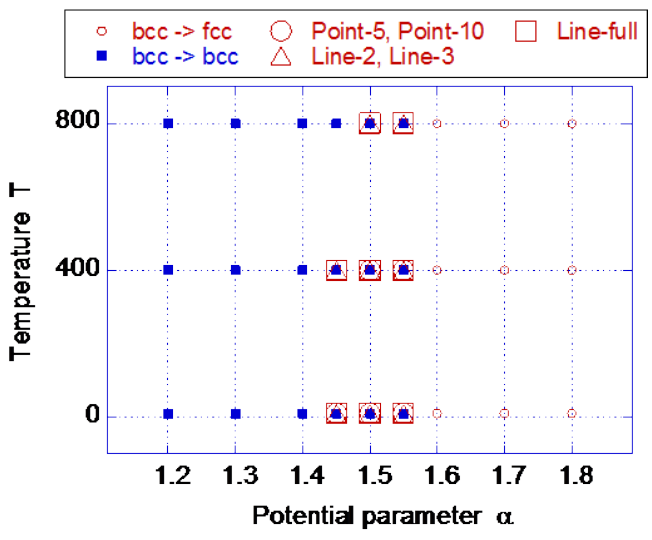

(a) Phase diagram

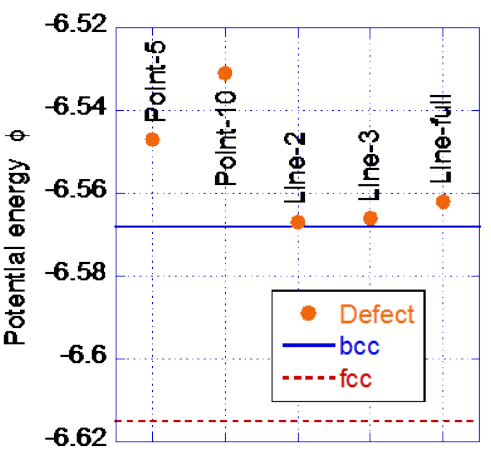

(b) Potential energy with defects

Figure 8. Phase diagram modified by defect models and potential energy with lattice defects for $\alpha=1.40$ and $T=10 \mathrm{~K}$.

higher values than that for line-defect models, but no specific effects on the phase transformation was found. Therefore, the initiation of the transformation is considered to originate in a local behavior, which is discussed in the next section.

\subsection{Plastic Behavior during Phase Transformation}

According to both the Bain and NW relationships, the bcc-to-fcc transformation is an overall phenomenon, involving changes in the edge length and/or simultaneous sliding of the atomic layer. Therefore, if no local irregularity exists, phase transformation progresses homogeneously. However, defects may induce local irregular behavior; in some of the simulations, notable patterns, which may be related to plastic deformation, were observed.

Figure 9 represents the variation in atomic arrangement during phase transformation obtained for a bcc-(001) model, Line-3 defect, $\alpha=1.55$, and $T=10 \mathrm{~K}$. Parts (a) and (b) show a snapshot on the $z-y$ plane and a 3-D view, respectively. Note that the lined efect is on the $x-y$ plane, part (a) corresponds to the side view, and every opposing face is connected through a periodic boundary. The color indicates the potential energy of each atom; the atoms on the regular lattice points are colored light blue, and the atoms at the irregularly arranged sites appearin different colors.

The phase transformation is initiated around the 1500th time step, and then a circular pattern is generated on the $z-y$ plane, as shown in Figure 9(a)(iii). This domain is cylindrical, as shown in the $3-\mathrm{D}$ view, and it grows larger as time passes. Subsequently, the cylindrical faces touch one another at about the 3500th time step, and the domain coalesces. Finally, two domains separated by parallel domain boundaries are generated. Interestingly, the origin of the cylindrical domain formation is not the defect itself but is separated from the defect.

Another notable pattern is shown in Figure 10, which also shows results for the bcc-(001) model, Line-3 defect, and $T=10 \mathrm{~K}$, with $\alpha=1.50$. Phase transformation 


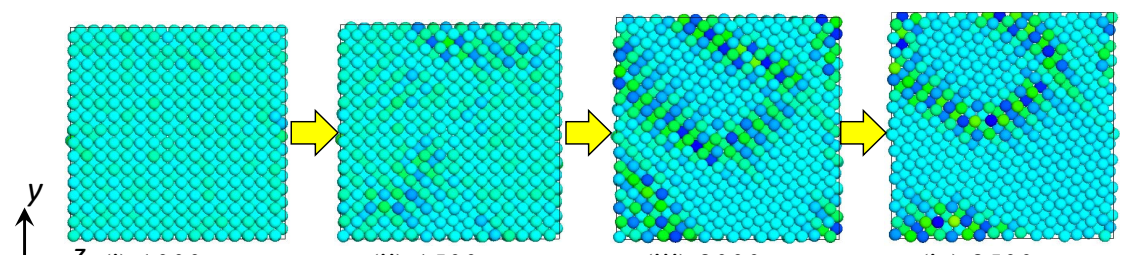

Z (i) 1000 step

(ii) 1500 step

(iii) 2000step

(iv) 2500 step

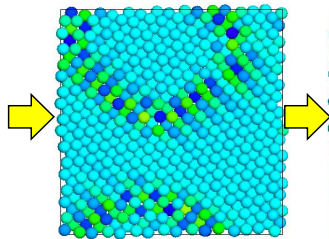

(v) 3000step
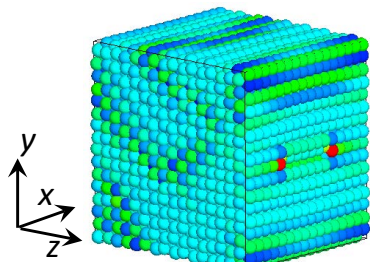

(i) 2000 step

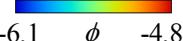

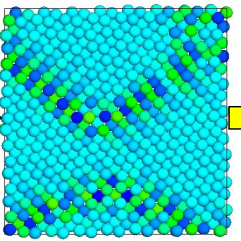

(vi) 3500step

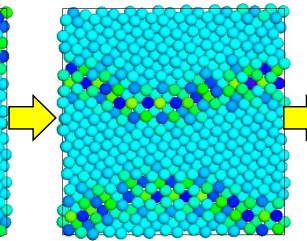

(vii) 4000step

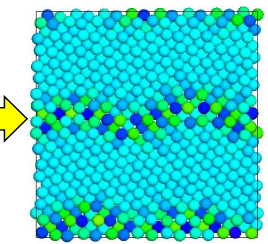

(viii) 5000step

(a) $x$-y plane

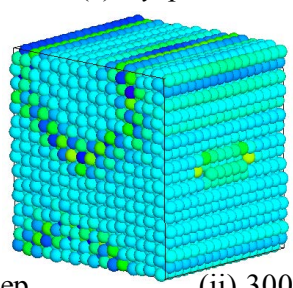

(b) 3-D view

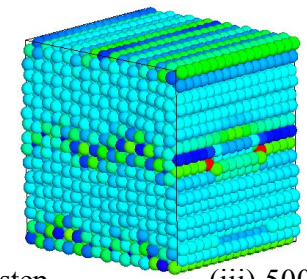

(iii) 5000 step

(ii) 3000step

Figure 9. Initiation of plastic behavior observed in transformation process with the bcc-(001) model, Line- 3 defect, $\alpha=1.55$, and $T=10 \mathrm{~K}$.

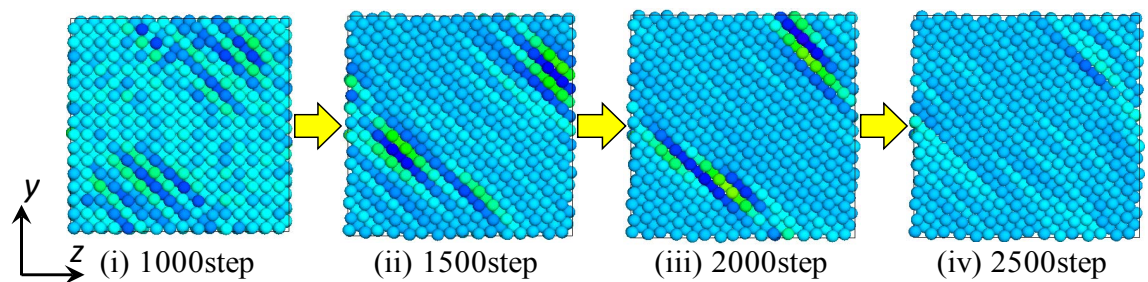

(a) $z-y$ plane

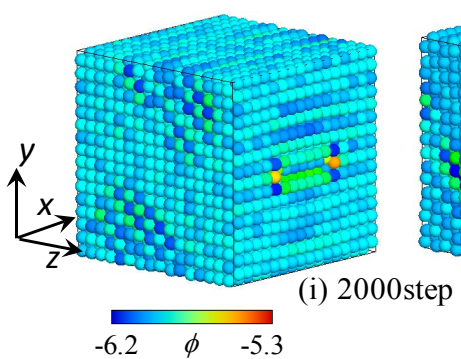

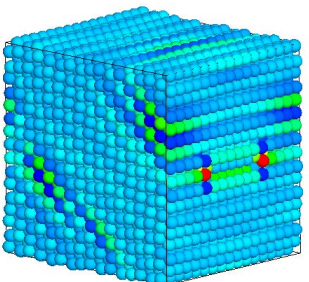

(ii) 3000 step

(b) 3-D view (iv) 2500step

Figure 10. Initiation of plastic behavior observed in transformation process with the bcc-(001) model, Line-3 defect, and $T=10 \mathrm{~K}$, with $\alpha=1.50$.

is initiated at about the 1500th timestep, which can be observed from the differences in the atomic configuration between the 1000th and 1500th time steps. Proceeding to the transformation, faint oblique lines are observed at the 1000th time step. These become sharper with the transformation at the 1500th time step and finally disappear when the transformation is completed by the 5000th time 
step. It is clear that the line corresponds to a plane in the 3-D view, and this is temporarily formed during the transformation. The origin of the plane can be tracked back to the defect, as seen in the 3-D view at the 3000th timestep, though this is ambiguous at the initiation period around the 1000th time step.

Similar behavior was observed for the full line models. Figure 11(a) represents the result for the same sets of parameters as for Figure 9, for which a circular pattern was observed. In this case, an arc can be seen at the 2000th timestep, as shown in Figure 11(a)(ii), but a closed circle is not formed, and two parallel boundaries are formed at the 3000th timestep. The origin of the generated plane can be found at the original defect, which is located at the center of the side edge in this figure. Similarly, the result of a comparative calculation using the same parameters as in Figure 10 is shown in Figure 11(b). In this case, the same pattern consisting of oblique sharp lines is observed. A minor difference is that the line does not disappear completely until the end, and the lines are partially retained.

\subsection{Discussion}

Overall, no specific patterns as shown in Figs 9 and 10 were observed during phase transformation for the scattered point defect models, although the total number of vacancies was much higher in a Point-10 model than that in a Line-3 model, and the increase in the potential energy was larger as shown in Figure 8(b). This is because heterogeneity is higher for aligned vacancies, or a line-defect model, than a randomly scattered case. Also, these patterns were not observed very frequently for the bcc-(011) models. This is because the Bain relationship induced often in the (011) model is rather straight forward, and local irregularities did not tend to be formed. In MD simulation, however, random inhomogeneity is introduced intrinsically, and different results were obtained occasionally. Figure 11(c) shows a result for the bcc-(011) Line-full model with $\alpha=1.50$ and $T=10 \mathrm{~K}$. A unique pattern with crossing lines is generated on the transformation between the 3000th and $4000^{\text {th }}$ time steps. Some other patterns were also observed, and detailed investigation of the universal features will continue.

\section{Conclusion}

Molecular dynamics simulations of the phase transformation from bcc to fcc structures were performed using a Morse-type potential, and the effects of lattice defects on the phase transformation were investigated. Consequently, it was revealed that the defects influenced the stable structure and accelerated transformation from bcc to fcc. Different types of lattice defects, such as cylindrical domain formation and planar defects, were also generated during phase transformation, which might result in the initiation of plastic deformation. Further investigation is necessary to clarify the relationship between the phase transformation and induced plastic deformation, and it is concluded that the validity of the 


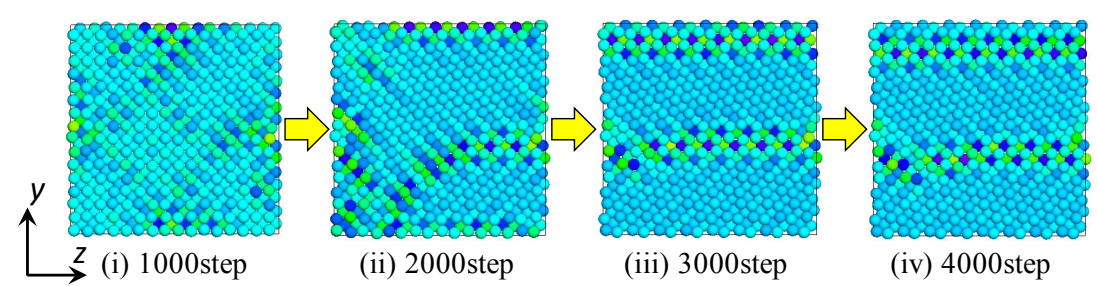

(a) bcc-(001), $\alpha=1.55, T=10 \mathrm{~K}$

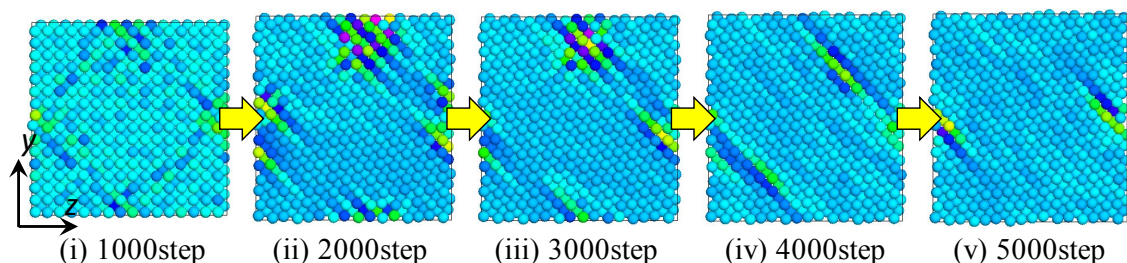

(b) bcc-(001), $\alpha=1.50, T=10 \mathrm{~K}$

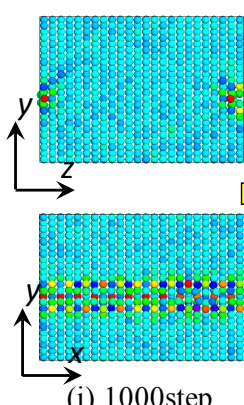

(i) 1000 step

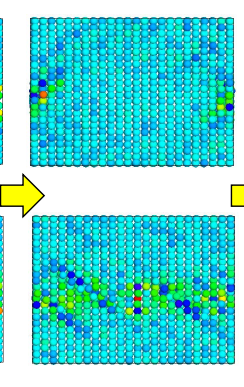

(ii) 2000step

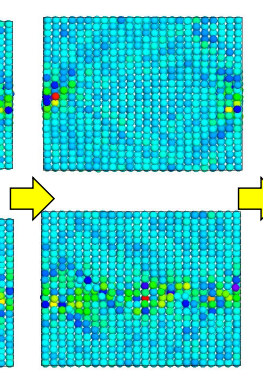

(iii) 3000 step

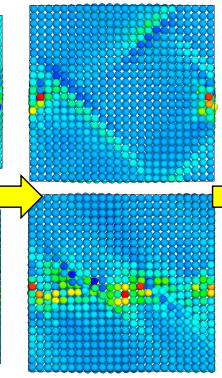

(iv) 4000 step

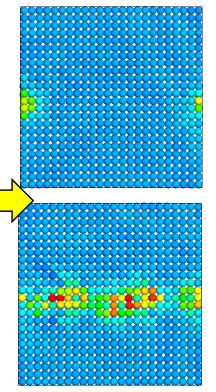

(v) 5000step

(c) bcc-(011), $\alpha=1.50, T=10 \mathrm{~K}$

Figure 11. Various patterns observed for the full-line models.

present model is verified. In addition, we have also reported microstructural change in polycrystalline material under severe plastic deformation [22] [23]. Very recently, an interesting study on the transformation mechanism between the bcc and fcc structures for a binary-alloy system was reported [24], where the transformation is initiated at the two-phase boundary and progresses based on the NW relationship. Further modeling combined with these models will lead to complete clarification of the transformation and induced plastic behavior.

\section{Conflicts of Interest}

The author declares no conflicts of interest regarding the publication of this paper.

\section{References}

[1] Bhadeshia, H.K.D.H. and honeycombe, R.W.K. (2017) Steels-Microstructure and properties. 4th Edition, Butterworth-Heinemann, Oxford.

[2] Greenwood, G.W. and Johnson, R.H. (1965) The Deformation of Metals under Small Stresses during Phase Transformations. Proceedings of the Royal Society of London. Series A, 283, 403-422. https://doi.org/10.1098/rspa.1965.0029

[3] Leblond, J.B., Devaux, J. and Devaux, J.C. (1989) Mathematical Modelling of 
Transformation Plasticity in Steels I: Case of Ideal-Plastic Phases. International Journal of Plasticity, 5, 551-572.

[4] Taleb, L. and Sidoroff, F. (2003) A Micromechanical Modeling of the Greenwood-Johnson Mechanism in Transformation Induced Plasticity. International Journal of Plasticity, 19, 1821-1842. https://doi.org/10.1016/S0749-6419(03)00020-2

[5] Inoue, T. (2007) Unified Transformation-Thermoplasticity and the Application. Journal of the Society of Materials Science Japan, 56, 352-356.

[6] Levitas, V.I. and Ozsoy, I.B. (2009) Micromechanical Modeling of Stress-Induced Phase Transformations. Part 1. Thermodynamics and Kinetics of Coupled Interface Propagation and Reorientation. International Journal of Plasticity, 25, 239-280. https://doi.org/10.1016/j.ijplas.2008.02.004

[7] Uehara, T. (2015) Molecular Dynamics Simulation on Transformation-Induced Plastic Deformation Using a Lennard-Jones Model. Key Engineering Materials, 626, 414-419. https://doi.org/10.4028/www.scientific.net/KEM.626.414

[8] Inoue, T., Ju, D.Y. and Arimoto, K. (1992) Metallo-Thermomechanical Simulation of Quenching Process-Theory and Implementation of Computer Code HEARTS. Proceedings of the 1 st International Conference on Quenching and the Control of Distortion, Chicago, IL, 22-25 September 1992, 205-212.

[9] Ju, D.Y., Mukai, R. and Sakamaki, T. (2011) Development and Application of Computer Simulation Code COSMAP on Induction Heat Treatment Process. International Heat Treatment and Surface Engineering, 5, 65-68. https://doi.org/10.1179/174951411X13051201040794

[10] Provatas, N. and Elder, K. (2010) Phase-Field Methods in Materials Science and Engineering. Wiley-VCH, Weinheim. https://doi.org/10.1002/9783527631520

[11] Schulz, S. (2016) Phase-Field Simulations of Multi-Component Solidification and Coarsening Based on Thermodynamic Datasets. KIT Scientific Publishing, Germany.

[12] Sekido, K., Ohmura, T., Hara, T. and Tsuzaki, K. (2012) Effect of Dislocation Density on the Initiation of Plastic Deformation on Fe-C Steels. Materials Transactions, 53, 907-912.

[13] Hata, K., Fujiwara, K., Kawano, K., Sugiyama, M., Fukuda, T. and Kakeshita, T. (2018) Three-Dimensional EBSD Analysis and TEM Observation for Interface Microstructure during Reverse Phase Transformation in Low Carbon Steels. ISIJ International, 58, 742-750.

[14] Engin, C. and Urbassek, H.M. (2008) Molecular-Dynamics Investigation of the FCC $\rightarrow$ BCC Phase Transformation in Fe. Computational Materials Science, 41, 297 304. https://doi.org/10.1016/j.commatsci.2007.04.019

[15] Tateyama, S., Shibuta, Y. and Suzuki, T. (2010) Orientation Relationship in FCCBCC Phase Transformation Kinetics of Iron: A Molecular Dynamics Study. ISIJ International, 50, 1211-1216.

[16] Li, G., Sui, X., Qin, X., Ma, Y., Wang, K. and Wang Q. (2016) Structural Transformation between BCC and FCC in Fe-Ni Nanoparticle during Heating Process. Physics Letters A, 380, 3500-3504. https://doi.org/10.1016/j.physleta.2016.08.019

[17] Ou, X. (2017) Molecular Dynamics Simulations of FCC-to-BCC Transformation in Pure Iron: A Review. Materials Science and Technology, 33, 822-835. https://doi.org/10.1080/02670836.2016.1204064

[18] Nguyen, T.Q., Sato, K. and Shibutani, Y. (2018) First-Principles Study of BCC/FCC Phase Transition Promoted by Interstitial Carbon in Iron. Materials Transactions, 
59, 870-875.

[19] Meiser, J. and Urbassek, H.M. (2018) Ferrite-to-Austenite and Austenite-to-Martensite Phase Transformations in the Vicinity of a Cementite Particle: A Molecular Dynamics Approach. Metals, 8, 837. https://doi.org/10.3390/met8100837

[20] Girifalco, L.A. and Weizer, V.G. (1959) Application of the Morse Potential Function to Cubic Metals. Physical Review, 114, 687-690. https://doi.org/10.1103/PhysRev.114.687

[21] Sandoval, L., Urbassek, H.M. and Entel, P. (2009) The Bain versus NishiyamaWassermann Path in the Martensitic Transformation of Fe. New Journal of Physics, 11, Article ID: 103027. https://doi.org/10.1088/1367-2630/11/10/103027

[22] Uehara, T. (2017) Molecular Dynamics Simulation of Grain Refinement in a Polycrystalline Material under Severe Compressive Deformation. Materials Sciences and Applications, 8, 918-932. https://doi.org/10.4236/msa.2017.812067

[23] Uehara, T. (2018) Molecular Dynamics Simulation of the Initiation of Plastic Deformation in Nanocrystalline Material. Proceedings of 9 th International Conference on Computational Methods, Rome, 6-10 August 2018, 700-705.

[24] Wei, W., Liu, L.C., Gong, H.R., Song, M., Chang, M.L. and Chen, D.C. (2019) Fundamental Mechanism of BCC-FCC Phase Transition from a Constructed PdCu Potential through Molecular Dynamics Simulation. Computational Materials Science, 159, 440-447. https://doi.org/10.1016/j.commatsci.2018.12.037 